\title{
Fortgeschrittenes Nierenzellkarzinom: Sunitinib bewährt sich im onkologischen Alltag
}

— Seit seiner Einführung ist der Tyrosinkinase-Inhibitor Sunitinib (Sutent ${ }^{\circledR}$ ) der Goldstandard für die Erstlinientherapie des metastasierten Nierenzellkarzinoms. Eine Beobachtungsstudie bestätigte nun den Stellenwert im klinischen Alltag. Im Expanded Access Programm erhielten weltweit 4.564 Patienten Sunitinib, darunter auch solche, die aus klinischen Studien aufgrund von Faktoren wie Alter, Metastasenlokalisation oder Tumorhistologie in der Regel ausgeschlossen werden, berichtete Dr. Edwin Herrmann, Münster. Im Zentrum der Studie standen Sicherheit, Therapiedauer und Ansprechraten.

Bei drei von vier Patienten konnte ein onkologischer Benefit erreicht werden: Bei 59\% stabilisierte sich die Erkrankung, bei $17 \%$ wurde eine partielle oder - selten - komplette Remission erreicht. Das progressionsfreie Überleben betrug knapp elf, das Gesamtüberleben rund 18 Monate. „Das entspricht nicht ganz dem, was wir in den Phase-III-Studien sehen“, so Herrmann. „Man muss aber bedenken, dass drei Viertel der Patienten bereits vorbehandelt waren." Bemerkenswert ist ferner, dass auch Subgruppen mit ungünstigem Profil - Alter über 65 Jahre, Hirnmetastasen, ECOG-PS ffl 2-vergleichbar gut auf die Behandlung ansprachen.

Nebenwirkungen fielen meist mild aus und bewegten sich im üblichen Rahmen: Diarrhöen, Müdigkeit und Übelkeit sowie von hämatologischer Seite Anämie, Thrombo- oder Neutropenie traten am häufigsten oft. Die Subgruppen unterschieden sich dabei nicht von der Gesamtstudienpopulation.

ara

Pressekonferenz „Standard of Care beim metastasierten Nierenzellkarzinom: Evidenz, Leitlinien und klinischer Alltag 2010" im Rahmen des 28. Deutschen Krebskongresses. Berlin, 26. Februar 2010 Veranstalter: Pfizer, Berlin

\section{Harninkontinenz: neues Generikum auf dem Markt}

— Die STADApharm GmbH hat zum 1. April 2010 das anticholinerge Blasenspasmolytikum Propiverin- $\mathrm{HCl}$ zur Behandlung von Harninkontinenz auf den Markt gebracht. Propiverin-HCI STADA enthält den Wirkstoff Propiverin (als Hydrochlorid, $\mathrm{HCl}$ ), der zu Wirkstoffklasse der Anticholinergika mit zusätzlicher kalziumantagonistischer Wirkkomponente gehört. Propiverin hemmt muskarinerge Rezeptoren der Blasenwand und senkt zusätzlich die Kontraktionskraft der Blasenwandmuskelzellen durch direkte Hemmung des Kalziumeinstroms in die Zelle. Die Indikationen von Propiverion- $\mathrm{HCl}$ STADA umfassen die Behandlung von Harninkontinenz und/oder erhöhtem Harndrang (häufiger Drang zum Wasserlassen) bei Patienten mit überaktiver Blase (angeboren oder durch Rückenmarkschädigung erworben).

Propiverin ist schnell bioverfügbar, wird in der Leber verstoffwechselt und hauptsächlich über die Nieren ausgeschieden. Bei schweren Nieren- und Leberfunktionsstö- rungen darf es nicht angewendet werden. Mögliche Nebenwirkungen sind unter anderem Mundtrockenheit, Sehstörungen und Verstopfung. Bei Überdosierung kann es zu zentralnervösen Wirkungen kommen.

Propiverin-HCI STADA Filmtabletten gibt es in den Wirkstärken $5 \mathrm{mg}$ und $15 \mathrm{mg}$. Die 5-mg-Filmtablette ist für die Anwendung bei Kindern und Erwachsenen mit einem Körpergewicht unter $35 \mathrm{~kg}$ vorgesehen.

STADA bietet Ärzten als besonderen Patienten-Service eine DVD mit Übungen zur Kräftigung der Beckenboden-Muskulatur an. Am Beispiel einfacher Übungen zeigt die DVD, wie man in wenigen Wochen die Beckenmuskulatur stärken kann. Der STADA-Sonder-Service fördert die Compliance, hilft Therapiekosten zu dämpfen und unterstützt die Behandlung der Harninkontinenz. Die DVD kann online bestellt werden unter www.stada.de (Fachkreise).

Nach Informationen von

Stada, Bad Vilbel
TURP: Aktuelle Informationen in der „TURP-Fibel“

Trotz der diversen Laser-Techniken ist die Transurethrale Elektroresektion der Prostata (TURP) Standard in der operativen Therapie der benignen Prostatahyperplasie (BPH). Prof. Peter Faul und Günter Farin haben mit Unterstützung durch FarcoPharma alles Wissenswerte in der TURP-Fibel zusammengefasst. Während Faul die klinischen Aspekte beleuchtet, erläutert Farin die physikalisch-technischen Grundlagen.

Die reich bebilderte Fibel richtet sich an alle interessierten Urologen, welche die TURP erlernen oder bereits praktizieren und ihre Kenntnisse vertiefen möchten. Sie ist ebenfalls hilfreich für urologisches Assistenzpersonal. Kostenlose Exemplare der TURP-Fibel sind unter info@farco-pharma.de oderwww. farco-pharma.de erhältlich.

Nach Informationen von Farco-Pharma, Köln

\section{Hilfe bei benigner Prostatahyperplasie}

Fast zwei Drittel aller Männer in der zweiten Lebenshälfte leiden unter einer benignen Prostatahyperplasie (BPH). Sandoz unterstützt die Behandlung mit qualitativen Arzneimitteln und Informationsmaterial.

Im Rahmen der medikamentösen Behandlung stehen bewährte apothekenpflichtige Urologika auf pflanzlicher Basis zur Verfügung. Gerade Phytotherapeutika wie Azuprostat $^{\circledR}$ Sandoz $^{\circledR}$ mit hochdosiertem Phytosterol $(65 \mathrm{mg})$ sowie Sabal Sandoz ${ }^{\circledR}$ und Urtica Sandoz ${ }^{\circledR}$ können unterstützend für die patientenindividuelle Therapie einer $\mathrm{BPH}$ eingesetzt werden.

Die Patientenbroschüre „Die Prostata, das unbekannte Wesen?" bietet für Betroffene klare Informationen über Thematik, Untersuchungen und Behandlungsoptionen und sensibilisiert zudem für die notwendigen Vorsorgeuntersuchungen. Die Broschüre ist über den Sandoz-Außendienst sowie unter www.sandoz. de (Service/Broschüren) erhältlich.

Nach Informationen von

Sandoz, Holzkirchen 